\title{
Interpreting core-level spectra of oxidizing phosphorene: Theory and experiment
}

\author{
Teng Yang, ${ }^{1}$ Baojuan Dong, ${ }^{1}$ Jizhang Wang, ${ }^{1}$ Zhidong Zhang, ${ }^{1}$ Jie Guan, ${ }^{1,2}$ Kaci Kuntz, ${ }^{3}$ \\ Scott C. Warren, ${ }^{4}$ and David Tománek ${ }^{1,2, *}$ \\ ${ }^{1}$ Shenyang National Laboratory for Materials Science, Institute of Metal Research, Chinese Academy of Sciences, \\ Shenyang 110016, People's Republic of China \\ ${ }^{2}$ Physics and Astronomy Department, Michigan State University, East Lansing, Michigan 48824, USA \\ ${ }^{3}$ Department of Chemistry, University of North Carolina at Chapel Hill, Chapel Hill, North Carolina 27599, USA \\ ${ }^{4}$ Department of Chemistry and Department of Applied Physical Sciences, University of North Carolina at Chapel Hill, \\ Chapel Hill, North Carolina 27599, USA
}

(Received 28 May 2015; revised manuscript received 18 August 2015; published 10 September 2015)

\begin{abstract}
We combine $a b$ initio density functional theory calculations with the equivalent cores approximation to determine core-level binding-energy shifts at phosphorus sites caused by oxidation of phosphorene. We find that presence of oxygen increases the core-level binding energies of $\mathrm{P}$ atoms and expect binding-energy shifts of up to $6 \mathrm{eV}$ in highly defective geometries. We have identified likely binding geometries of oxygen that help to interpret the observed core-level photoemission spectra in samples at different stages of oxidation and allow us to determine the fractions of specific local geometries.
\end{abstract}

DOI: 10.1103/PhysRevB.92.125412

PACS number(s): 73.20.At, 73.61.Cw, 61.46.-w, 73.22.-f

\section{INTRODUCTION}

Phosphorene, a monolayer of black phosphorus, has attracted significant attention recently due to its robust direct fundamental band gap, high carrier mobility, and a large density of states outside the band-gap region, making it a likely candidate for two-dimensional (2D) electronics [1-4]. The fundamental drawback of elemental phosphorus is its chemical reactivity, which poses a significant challenge [5,6]. Whereas most experimental data have been obtained using mechanically exfoliated few-layer phosphorene [1-4,7], liquid exfoliation seems to offer a more scalable alternative $[8,9]$. In all cases, exposure of phosphorene to air is known to oxidize the samples, degrading their electronic properties [10]. The precise oxidation mechanism and the atomic structure of the oxidized sample are largely unknown, since oxidation leads to increasingly amorphous structures that cannot be probed by diffraction, and since most techniques that probe the directspace structure lead to further sample degradation. Theoretical studies of defective or oxidized phosphorene provide possible geometries [11-13], but it is unclear if such geometries are present in real samples. Spectroscopic techniques, including $\mathrm{x}$-ray photoemission spectroscopy (XPS) in the core-level region, are indicative of changes in the extent of oxidation, but have not-so far-provided detailed insight into the local chemical structure.

Here we combine $a b$ initio density functional theory (DFT) calculations with the equivalent cores approximation to determine core-level binding-energy shifts at phosphorus sites caused by oxidation of phosphorene. We find that presence of oxygen increases the core-level binding energies of $\mathrm{P}$ atoms by up to $6 \mathrm{eV}$ in highly defective geometries. We have identified likely binding geometries of oxygen that help to interpret the observed core-level photoemission spectra in samples at different stages of oxidation and allow us to determine the fractions of specific local geometries.

*tomanek@pa.msu.edu

\section{COMPUTATIONAL TECHNIQUES}

In order to gain insight into possible oxidized sites and corelevel electronic binding-energy shifts of phosphorus atoms, we used $a b$ initio density functional theory as implemented in the VASP code [14] to model phosphorene and related phosphorus oxide structures. We used periodic boundary conditions with monolayer structures represented by a periodic array of slabs separated by a vacuum region in excess of $15 \AA$. Similarly, finite-size fragments have been represented by a periodic array of quantum dots separated by a vacuum region of $15 \AA$. We used the projector-augmented-wave pseudopotentials [15] and the Perdew-Burke-Ernzerhof [16] exchange-correlation functional. The Brillouin zone of the primitive unit cell of the $2 \mathrm{D}$ structures was sampled by $9 \times 9 \times 1 k$ points and that of the $2 \times 3$ superlattice was sampled by $5 \times 5 \times 1 k$ points [17]. We used $500 \mathrm{eV}$ as the electronic kinetic-energy cutoff for the plane-wave basis and a total-energy difference between subsequent self-consistency iterations below $10^{-6} \mathrm{eV}$ as the criterion for reaching self-consistency. Net charges on atoms have been estimated using the Bader population analysis [18]. All geometries have been optimized using the conjugate-gradient method [19], until none of the residual Hellmann-Feynman forces exceeded $10^{-2} \mathrm{eV} / \AA$.

\section{EXPERIMENTAL TECHNIQUES}

Few-layer phosphorene samples were prepared using liquid exfoliation and fractionated by centrifugation [8]. The resulting solutions contained flakes of thicknesses ranging from one to seven layers. Thin films of few-layer phosphorene were doctor bladed onto gold-coated silicon wafers and exposed to three controlled environments for $18 \mathrm{~h}$ : (1) nitrogen ( $\leqslant 1 \mathrm{ppm}$ $\mathrm{O}_{2}$ ), (2) $99.999 \%$ pure oxygen, and (3) ambient conditions (air, ca. 50\% humidity). Exposure to oxygen and air was accompanied by irradiation with blue light $(460 \mathrm{~nm})$ from a light-emitting diode at an intensity of $0.6 \mathrm{~mW} / \mathrm{cm}^{2}$. XPS was performed using a Kratos Axis Ultra Delay-Line Detector (DLD) spectrometer with monochromatic $\mathrm{Al} \mathrm{K}_{\alpha}$ source. 
(a)

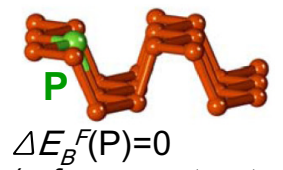

(reference structure)

(c)

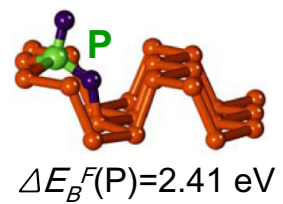

(e)

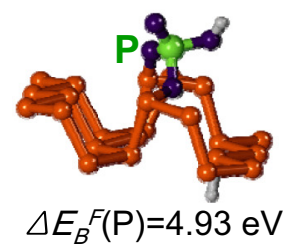

(g)

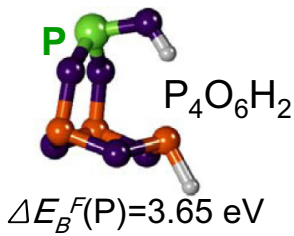

(i)

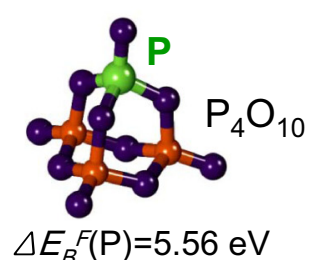

(b)

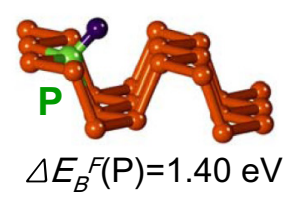

(d)

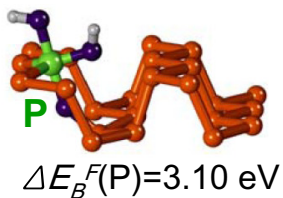

(f)

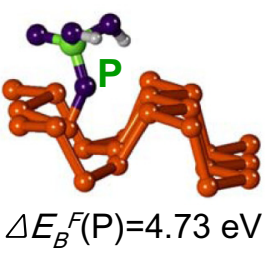

(h)

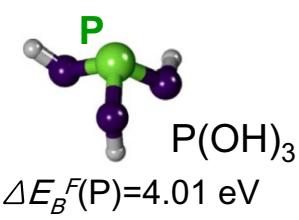

(j)

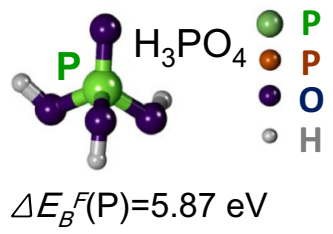

FIG. 1. (Color online) Ball-and-stick models of selected pristine, defective, and oxidized phosphorene structures. Structures (a)-(f) represent a defect in a phosphorene monolayer, and (g)-(j) are isolated fragments. The phosphorus atom, for which core-level binding-energy shifts are calculated, is highlighted in light green. Calculated core-level binding-energy shifts $\Delta E_{B}^{F}(\mathrm{P})$ for this specific $\mathrm{P}$ atom are given below each structure.

\section{RESULTS}

\section{A. Equilibrium structures}

We have systematically examined all possible combinations of $\mathrm{P}$ with $-\mathrm{P},-\mathrm{OP},-\mathrm{OH}$, and $=\mathrm{O}$ substituents. Because neutrally charged $\mathrm{P}$ is typically limited to oxidation states of 3 or 5 and bond orders of 3 or 5 [20], we have ensured that the combinations we generated follow this rule. In addition, we consider a structure predicted earlier [11] in which phosphorene is functionalized with $=\mathrm{O}$, yielding an unconventional oxidation state of 2 and bond order of 5 . We present some of the optimized geometries in Fig. 1. Many of these structures are derived from the most stable allotrope, black phosphorus [21], also known as $\alpha$-P, and some are molecular fragments. Since oxidation is a nonequilibrium process, the occurrence of a particular structure is not necessarily related to its stability. The equilibrium structure of a pristine phosphorene monolayer, with all P sites being locally equivalent, is shown in Fig. 1(a).

Defective structures, presented in other subfigures of Fig. 1, contain $\mathrm{P}$ atoms that have different chemical environments. Changing the chemical environment changes the local potential at a given $\mathrm{P}$ site, altering its core-level binding energy. In the following, we estimate the core-level binding-energy shifts $\Delta E_{B}^{F}(\mathrm{P})$ at different $\mathrm{P}$ sites in defective samples with respect to the value $E_{B}^{F}(\mathrm{P}$ in $\alpha-\mathrm{P})$ found in a pristine $\alpha-\mathrm{P}$ monolayer.

\section{B. The Born-Haber cycle and the equivalent cores approximation}

$\mathrm{X}$-ray photoemission spectroscopy measures the kineticenergy distribution of photoelectrons, amounting to the energy $h v$ of the incident photon reduced by the core-level binding energy $E_{B}^{F}$ with respect to the Fermi level and the work function of the sample. The core-level binding energy can be determined by the difference between the total energy of the system in the final state, which contains a core hole in the atom of interest, and the total energy of the unperturbed system in the initial state. Even though the core-level binding energy could be determined directly in this way using an all-electron calculation, the necessary approximations would significantly reduce the reliability of the result.

Since we are primarily interested not in the absolute value of the core-level binding energy but rather in the energy shift caused by changes in the chemical environment, we use a thermodynamic Born-Haber cycle as a viable alternative to an all-electron total-energy calculation. The benefit of this approach, which is depicted in Fig. 2 and discussed in the following, is improved accuracy and additional insight into the physics behind core-level binding-energy shifts.

The usefulness of considering a thermodynamic cycle to describe core-level binding energies results from decoupling total-energy changes caused by different chemical environments from electronic excitation energies in isolated atoms. The former processes reflect cohesion of the crystal or chemisorption energies, which can be described accurately using DFT with valence electrons only. The latter processes are independent of the chemical environment and cancel when considering energy shifts only. The Born-Haber cycle makes use of the "equivalent cores" or the " $Z+1$ " approximation [22], which claims that in an isolated atom the effect of a core hole on the valence electrons is identical to adding a proton to the nucleus. As we will discuss later on, the additional assumption of a fully screened final state is satisfied in the experiment. The final-state screening occurs in the valence region by an electron at the Fermi level; the subsequent filling of the core hole occurs much later and does not affect the kinetic energy of the emitted electron. This elegant approach has been used successfully to interpret the origin of core-level binding-energy shifts at different transition-metal surfaces [23] and during chemisorption processes [24,25] including the catalytic formation of $\mathrm{NH}_{3}$ from $\mathrm{N}_{2}$ and $\mathrm{H}_{2}$.

The thermodynamic Born-Haber cycle, which determines the core-level binding energy at a particular $\mathrm{P}$ site in pristine phosphorene by bridging the energy difference between the initial and the final state, is depicted in Fig. 2(a). The initial state contains the $\mathrm{P}$ atom of interest, highlighted by the light green color in Fig. 1(a), in its electronic ground state. Next, this atom is separated from its environment, which requires 


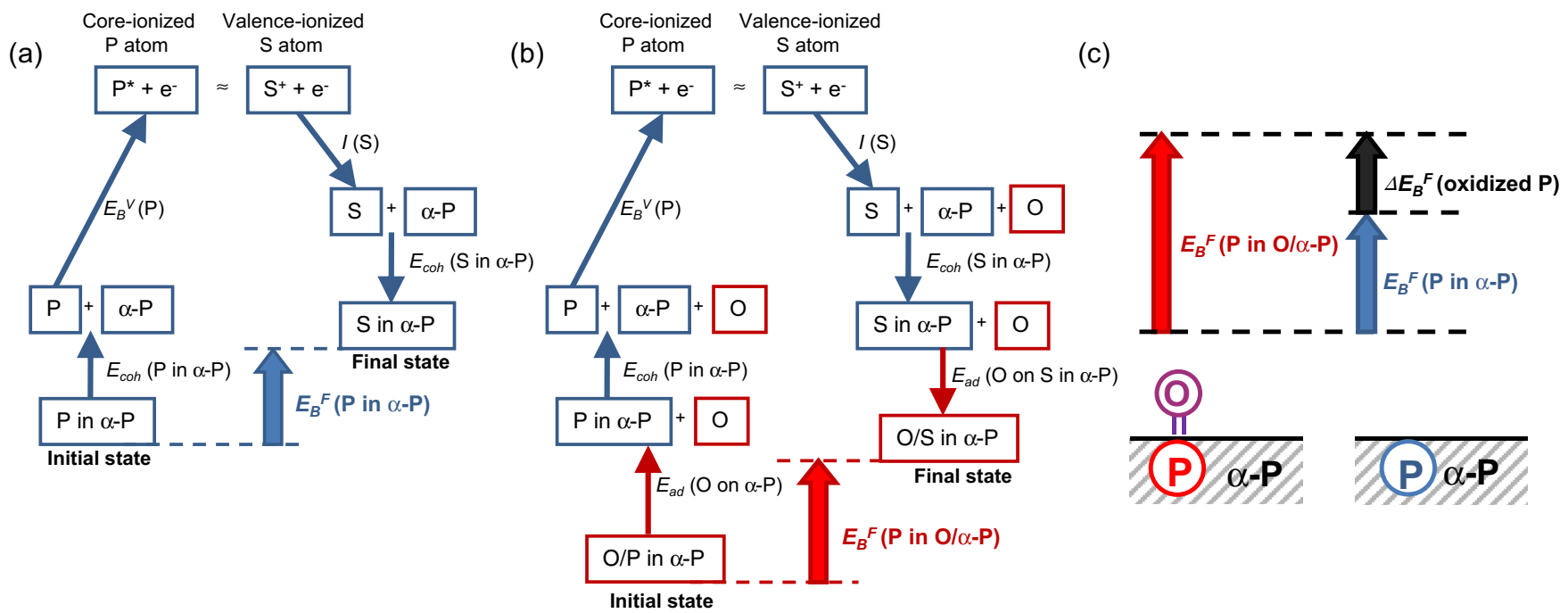

FIG. 2. (Color online) Schematic of the thermodynamic Born-Haber cycle used to determine core-level binding energies of $\mathrm{P}$ atoms in (a) pristine and (b) oxidized phosphorene. (c) Schematic illustration of the cause of the core-level binding-energy shift $\Delta E_{B}^{F}$ (oxidized P).

the investment of the binding energy $E_{\text {coh }}(\mathrm{P}$ in $\alpha-\mathrm{P})$. In the following step, a core electron in this isolated $\mathrm{P}$ atom is photoexcited to the vacuum level, requiring the energy $E_{B}^{v}$. In the $Z+1$ approximation, the core-ionized $\mathrm{P}^{*}$ ion is considered equivalent to a valence-ionized $\mathrm{S}^{+}$ion. Assuming that the final state is fully screened, the isolated $\mathrm{S}^{+}$ion will recombine with the free valence electron, releasing the ionization potential energy $I(\mathrm{~S})$. Finally, the $\mathrm{S}$ atom will be implanted as an impurity at the site of the reference $\mathrm{P}$ atom, releasing the binding energy of an S impurity in $\alpha$-P. Summing up the energies associated with the individual steps then yields the energy difference between the final and the initial state, which corresponds to the core-level binding energy $E_{B}^{F}(\mathrm{P}$ in $\alpha-\mathrm{P})$ of a phosphorus atom in phosphorene.

As an example of a modified system, we consider an oxygen atom bonded to the $\mathrm{P}$ atom of interest, shown in Fig. 1(b). The Born-Haber cycle for this $\mathrm{P}$ atom in oxidized phosphorene, shown in Fig. 2(b), closely resembles that for a $\mathrm{P}$ atom in pristine phosphorene in Fig. 2(a). The only difference between the two cycles involves the desorption energy $E_{\mathrm{ad}}(\mathrm{O}$ on $\alpha-\mathrm{P})$ of the $\mathrm{O}$ atom from phosphorene in the initial state and the adsorption energy $E_{\text {ad }}(\mathrm{O}$ on $\mathrm{S}$ in $\alpha-\mathrm{P})$ of the $\mathrm{O}$ atom on a sulfur impurity in phosphorene in the final state. Clearly, such a cycle can be constructed for any other type of defect in phosphorene.

The core-level binding-energy shift, shown schematically in Fig. 2(c), is defined by

$$
\Delta E_{B}^{F}(\text { oxidized } \mathrm{P})=E_{B}^{F}(\mathrm{P} \text { in } \mathrm{O} / \alpha-\mathrm{P})-E_{B}^{F}(\mathrm{P} \text { in } \alpha-\mathrm{P}) .
$$

As can be inferred from the comparison of Figs. 2(a) and 2(b), the energy associated with the electronic rearrangement in isolated atoms drops out in the difference. This involves in particular the assumption implied in the equivalent cores approximation that the total energy of an isolated core-ionized $\mathrm{P}^{*}$ ion should equal that of a valence-ionized $\mathrm{S}^{+}$ion. Then,

$$
\Delta E_{B}^{F}(\text { oxidized } \mathrm{P})=E_{\mathrm{ad}}(\mathrm{O} \text { on } \alpha-\mathrm{P})-E_{\mathrm{ad}}(\mathrm{O} \text { on } \mathrm{S} \text { in } \alpha-\mathrm{P}),
$$

namely, the difference between the adsorption energy of an oxygen atom on a sulfur impurity in $\alpha$-P and on a phosphorus atom in pristine $\alpha$-P. Thus, the binding-energy shift in the core-level region caused by changes in the crystal potential can be related to chemical reaction energies in the electronic ground state. These chemical reaction energies can be calculated accurately by DFT with valence electrons only.

For defects other than a single chemisorbed oxygen atom, we need to replace the oxygen adsorption energy $E_{\mathrm{ad}}(\mathrm{O})$ by the energy difference between the defective and the ideal structure both in the initial and the final state. In all cases, the local environment structure near the sulfur atom in the final state must be the same as that of the equivalent $\mathrm{P}$ atom in the initial state, since atomic relaxations caused by elemental substitution would occur on a much longer time scale than electronic excitations.

\section{Calculated core-level binding-energy shifts}

The calculated core-level binding-energy shifts for the highlighted phosphorus atoms in different structures are shown below each structure in Fig. 1. As seen in Fig. 1(b), chemisorption of an oxygen atom increases the core-level binding energy of a phosphorus atom in $\alpha$-phosphorene by $1.4 \mathrm{eV}$. Much larger core-level binding energy shifts are obtained for other structures, the largest values of up to $6 \mathrm{eV}$ occurring for a $\mathrm{P}$ atom in phosphoric acid, $\mathrm{H}_{3} \mathrm{PO}_{4}$, and phosphorus oxide, $\mathrm{P}_{4} \mathrm{O}_{10}$. Consequently, the presence of such species should increase the core-level binding energies of phosphorus atoms by $1.4-6 \mathrm{eV}$, thus elucidating the cause of a new broad peak in the range of $131-136 \mathrm{eV}$ in the observed core-level spectra shown in Fig. 3.

Since it is a priori not clear to what degree a functional group attached to a particular phosphorus atom also modifies the core-level binding energies at the neighboring phosphorus sites, we performed the corresponding calculations for the geometries in Figs. 1(b)-1(d). Our results, shown in Fig. 4, indicate that core-level binding-energy shifts $\left|\Delta E_{B}^{F}\right| \gtrsim 1 \mathrm{eV}$ 


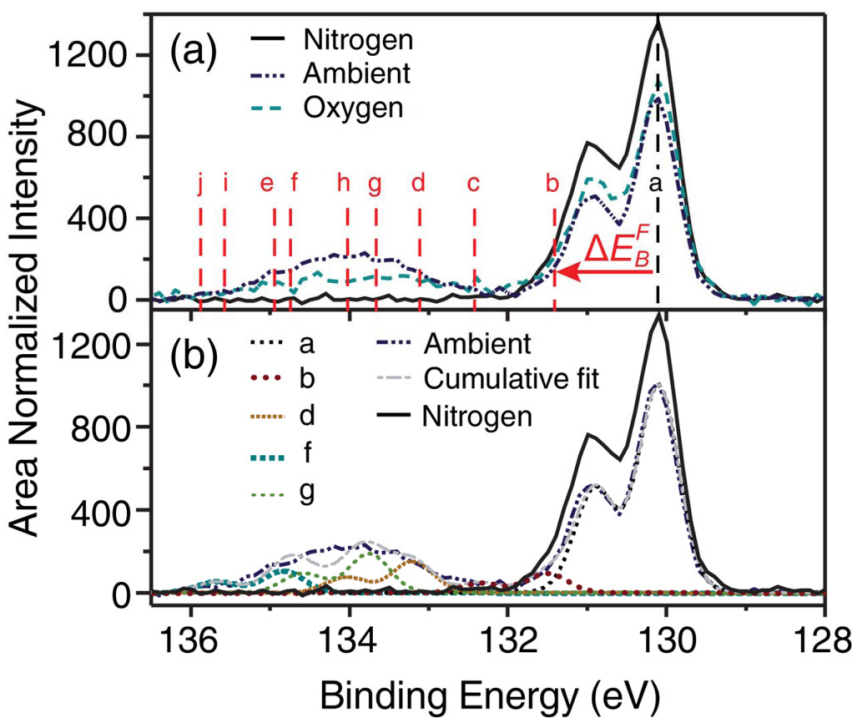

FIG. 3. (Color online) Phosphorus core-level spectra in fewlayer phosphorene at different levels of oxidation. (a) Observed spectra of pristine samples under inert $\mathrm{N}_{2}$ atmosphere, given by the solid black line, are compared to spectra obtained after exposure to ambient conditions, shown by the dot-dashed blue line, or pure oxygen, shown by the dashed green line. Expected positions of the $2 p_{3 / 2}$ peak of $\mathrm{P}$ in different chemical environments, specified in Fig. 1, are shown by the vertical dashed lines. $\Delta E_{B}^{F}$ denotes the core-level binding-energy shift for a particular geometry. (b) Spectral decomposition of the feature in the 132-136-eV binding-energy range in the sample exposed to ambient conditions into components attributed to different structures. The contribution of representative functional groups is described by rigidly shifting the spectrum of the pristine species. (a)

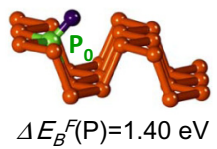

(d)

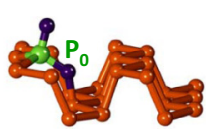

$\Delta E_{B} F(\mathrm{P})=2.41 \mathrm{eV}$

(g)

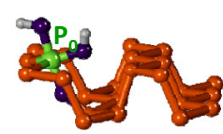

$\Delta E_{B} F(\mathrm{P})=3.10 \mathrm{eV}$ (b)

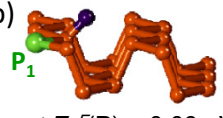

$\Delta E_{B} F(\mathrm{P})=-0.03 \mathrm{eV}$

(e)

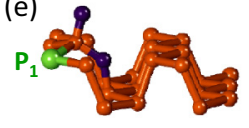

$\Delta E_{B}^{F}(\mathrm{P})=0.05 \mathrm{eV}$

(h)

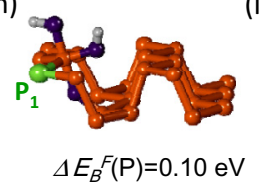

(f) (c)
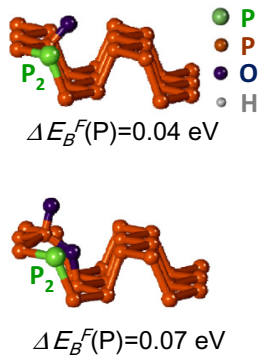

(i)

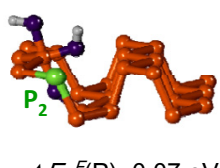

FIG. 4. (Color online) Calculated core-level binding-energy shifts $\Delta E_{B}^{F}$ at different phosphorus sites in oxidized phosphorene. Structures (a)-(c) are identical to the structure in Fig. 1(b), structures (d)-(f) are identical to the structure in Fig. 1(c), and structures (g)-(i) are identical to the structure in Fig. 1(d). $\mathrm{P}_{0}, \mathrm{P}_{1}$, and $\mathrm{P}_{2}$ indicate the specific phosphorus sites, for which the core-level binding-energy shifts are being calculated. are expected only for sites labeled $\mathrm{P}_{0}$ that are directly connected to the functional group. Core-level binding-energy shifts at first- and second-neighbor sites, labeled $\mathrm{P}_{1}$ and $\mathrm{P}_{2}$ in Fig. 4, are all in the range $\left|\Delta E_{B}^{F}\right| \lesssim 0.1 \mathrm{eV}$. Due to the finite resolution of the XPS spectra, these sites cannot be distinguished from pristine phosphorus.

To understand the differences among the core-level binding energies, we compare the local chemical environment of the photo-excited phosphorus atoms in Fig. 1 that modifies the crystal potential at that site. To help in the interpretation of core-level shifts, we also determine the Bader charge $Q(\mathrm{P})$ at the phosphorus site of interest. In general, we find that the corelevel binding energy increases with increasing net positive Bader charge on the $\mathrm{P}$ atom of interest. It is reassuring that the calculated Bader charges also correlate with the number and type of nearest neighbors, such as whether those neighbors are electron donating or withdrawing.

Because of the large number of possible phosphorus oxide geometries, we begin by sorting these structures into four groupings based on bond order and oxidation state, the latter of which correlates loosely with the Bader charge. The first group contains phosphorus atoms with a formal oxidation state of $2+$ and with a bond order of 5; there is a single structure, shown in Fig. 1(b), which falls in this category, and the predicted binding-energy shift is $1.4 \mathrm{eV}$. The second group, represented by the structures in Figs. 1(c) and 1(d), contains phosphorus atoms with a formal oxidation state of $3+$ and with a bond order of 5; we predict binding energy shifts of 2.0-3.1 eV. The third group, represented by the structures in Figs. 1(g) and 1(h), contains phosphorus atoms with a formal oxidation state of $3+$ and with a bond order of 3 ; we predict binding-energy shifts in the 3.5-4.0-eV range. The fourth group, represented by the structures in Figs. 1(e), 1(f), 1(i), and 1(j), contains phosphorus with a formal oxidation state of $5+$ and with a bond order of 5; we observe binding energy shifts in the $5-6-e V$ range. The relative magnitudes of these shifts follow general chemical intuition: the gradual replacement of electron donating substituents by electron withdrawing substituents, as shown in the progression from groups 1 to 4 , leads to a monotonic increase in binding energy. This intuitive picture is borne out by our calculations, which yield average Bader charges of $+1.1 e$ for group $1,+1.9 e$ for group $2,+2.2 e$ for group 3, and $+3.6 e$ for group 4 .

Interestingly, we also find that even for phosphorus atoms that have similar Bader charges, such as those in groups 2 and 3 , the shift in binding energy can differ by up to $2.0 \mathrm{eV}$. This underscores the influence that local geometry and the nature of bonding have on the observed binding energies. In general, we find that the binding energy is extremely sensitive to the identity of the nearest neighbors, only moderately sensitive to the identity of the second-nearest neighbors, and insensitive to the identity of the third-nearest neighbors. This finding is of central importance, because it allows the binding-energy shifts of Fig. 1 to be applied to phosphorus atoms in many types of molecular or extended structures so long as the local arrangement of first- and second-nearest neighbors is the same. Therefore, it is inconsequential that some of the structures of Fig. 1 are molecular, because the same local bonding motifs can be found within extended structures. Likewise, since the binding energy is sensitive to only the local environment, it 
does not matter whether the phosphorus oxides are amorphous or crystalline.

\section{XPS analysis of few-layer phosphorene binding energies}

In order to determine which phosphorus oxides are present in real samples, we used XPS to measure core-level binding energies in pristine and photo-oxidized few-layer phosphorene and present our results in Fig. 3. Comparing our XPS spectra with referenced $\mathrm{Au} 4 f$ and $\mathrm{C} 1 s$ binding energies indicated the absence of charging, allowing an accurate application of the Born-Haber cycle illustrated in Fig. 2.

The observed binding-energy values of $130.1 \mathrm{eV}$ for the $2 p_{3 / 2}$ and $131.0 \mathrm{eV}$ for the $2 p_{1 / 2}$ core electrons in few-layer phosphorene are within $0.0-0.6 \mathrm{eV}$ of values that have been reported previously for bulk black phosphorus [26-28]. The same core-level binding energies are also present in samples that have been exposed to air and oxygen, indicating that much of the phosphorus remains in the zero oxidation state. The emergence of peaks at higher binding energies, however, does indicate that some of the phosphorus has become oxidized. Indeed, the observation of binding energies in the 132-136-eV region is customarily assigned to various phosphorus oxides, although the structure and composition of these oxides are often unspecified [5,8,29]. We are confident that additional features in the core-level binding energy region are caused neither by plasmonic excitations nor by electronic rearrangements in the valence electron region. According to previous studies [30], the plasmon is found at $20 \mathrm{eV}$, which is significantly larger than the observed core-level binding-energy shifts in the 2-6-eV range and thus would not obscure the signal. Peaks corresponding to binding-energy shifts of 2-6 eV are also not attributable to valence excitations in the phosphorus oxides because these have large band gaps, typically exceeding $6 \mathrm{eV}$.

The clustering of binding-energy shifts into chemically intuitive groups defined in the previous subsection has allowed us to quantify the relative abundance of each type of structure through decomposition of the XPS spectra. First, we selected a single representative structure from each of the four groups; the chosen structure had a calculated binding-energy shift close to the average value observed for each grouping. Using the calculated binding-energy shifts of Fig. 1 for the four representative structures, we fit our area-normalized experimental spectra with a linear combination of Voigt functions while minimizing the residual error: each function was centered at the determined binding-energy shift and restricted to the same full width at half maximum value observed for the ground-state P, shown in Fig. 1, with a $2 p$ doublet at 130.1 and $131.0 \mathrm{eV}$.

The resulting decomposition of the XPS spectra allowed for the assessment of the relative quantities of phosphorus oxides with specific chemical structures. Results for the analysis of samples exposed to the ambient are presented in Fig. 3(b). In this sample, the percentage of oxidized phosphorus is $17.4 \%$ in group $1,28.1 \%$ in group $2,34.9 \%$ in group 3 , and $19.6 \%$ in group 4. In the oxygen-exposed sample, the percentages of oxidized phosphorus are $33.7 \%$ in group $1,29.1 \%$ in group 2 , $18.4 \%$ in group 3 , and $18.8 \%$ in group 4 .

Our strategy of organizing structures into representative groups provides insight into the amounts of each group present in oxidized phosphorus. It is important to note, however, that there is some uncertainty in these estimates, since several structures from each group may be present in experimental samples. In addition, some byproducts such as phosphoric acid may be volatile within the high vacuum of the XPS chamber and therefore may not be present in the XPS spectra. Nevertheless, our analysis does suggest that exposure to oxygen and water (ambient) does lead to higher degrees of oxidation than exposure to oxygen only. In fact, it has been suspected [6] that water plays an important role in the degradation of phosphorus and phosphorus oxides, and our results are consistent with this hypothesis.

\section{SUMMARY AND CONCLUSIONS}

In summary, we used $a b$ initio density functional theory calculations to determine likely defect structures in phosphorene exposed to ambient and oxygen-only conditions. Using the equivalent cores approximation, we determined core-level binding-energy shifts at defective phosphorus sites. We found that the presence of oxygen increased the core-level binding energies of $\mathrm{P}$ atoms and found binding-energy shifts of up to $6 \mathrm{eV}$ in highly defective geometries. These findings agree with core-level spectra observed by XPS. We have found that most phosphorus atoms at oxygen-induced defects are in the trivalent oxidation state with three to five neighboring $\mathrm{P}, \mathrm{O}$, or $\mathrm{H}$ atoms. We believe that only a small fraction of $\mathrm{P}(\mathrm{V})$ atoms is present in these samples, likely in the form of small molecules including phosphoric acid and phosphorus oxides.

\section{ACKNOWLEDGMENTS}

J.G. and D.T. appreciate the hospitality of the Institute of Metal Research (IMR), where this research has been conducted. T.Y. acknowledges the National Natural Science Foundation of China under Grant No. 51331006 and the Key Research Program of Chinese Academy of Sciences (Grant No. KGZD-EW-T06) for financial support. S.C.W. acknowledges start-up funds from University of North Carolina Chapel Hill. Z.Z.'s honorary authorship as division head is required by IMR institutional policy.
[1] L. Li, Y. Yu, G. Jun Ye, Q. Ge, X. Ou, H. Wu, D. Feng, X. H. Chen, and Y. Zhang, Black phosphorus field-effect transistors, Nature Nanotech. 9, 372 (2014).

[2] H. Liu, A. T. Neal, Z. Zhu, Z. Luo, X. Xu, D. Tomanek, and P. D. Ye, Phosphorene: An unexplored 2d semiconductor with a high hole mobility, ACS Nano 8, 4033 (2014).
[3] S. P. Koenig, R. A. Doganov, H. Schmidt, A. H. Castro Neto, and B. Özyilmaz, Electric Field Effect in Ultrathin Black Phosphorus, Appl. Phys. Lett. 104, 103106 (2014).

[4] T. Low, M. Engel, M. Steiner, and P. Avouris, Origin of photoresponse in black phosphorus phototransistors, Phys. Rev. B 90, 081408 (2014). 
[5] J. D. Wood, S. A. Wells, D. Jariwala, K.-S. Chen, E. K. Cho, V. K. Sangwan, X. Liu, L. J. Lauhon, T. J. Marks, and M. C. Hersam, Effective passivation of exfoliated black phosphorus transistors against ambient degradation, Nano Lett. 14, 6964 (2014).

[6] A. Favron, E. Gaufrès, F. Fossard, A.-L. Phaneuf-L'Heureux, N. Y.-W. Tang, P. L. Lévesque, A. Loiseau, R. Leonelli, S. Francoeur, and R. Martel, Photooxidation and quantum confinement effects in exfoliated black phosphorus, Nat. Mater. 14, 826 (2015).

[7] A. Favron, E. Gaufrès, F. Fossard, P. L. Lévesque, A.-L. Phaneuf-L'Heureux, N. Y.-W. Tang, A. Loiseau, R. Leonelli, S. Francoeur, and R. Martel, Exfoliating pristine black phosphorus down to the monolayer: Photo-oxidation and electronic confinement effects, arXiv:1408.0345.

[8] A. H. Woomer, T. W. Farnsworth, J. Hu, R. A. Wells, C. L. Donley, and S. C. Warren, Phosphorene: Synthesis, Scale-Up, and Quantitative Optical Spectroscopy, ACS Nano, Article ASAP.

[9] J. Kang, J. D. Wood, S. A. Wells, J.-H. Lee, X. Liu, K.-S. Chen, and M. C. Hersam, Solvent exfoliation of electronic-grade, twodimensional black phosphorus, ACS Nano 9, 3596 (2015).

[10] D. Tománek, 2014 symposium on phosphorene: An emerging 2D semiconductor, Mater. Express 4, 545 (2014).

[11] A. Ziletti, A. Carvalho, D. K. Campbell, D. F. Coker, and A. H. Castro Neto, Oxygen Defects in Phosphorene, Phys. Rev. Lett. 114, 046801 (2015).

[12] A. Ziletti, A. Carvalho, P. E. Trevisanutto, D. K. Campbell, D. F. Coker, and A. H. Castro Neto, Phosphorene oxides: Bandgap engineering of phosphorene by oxidation, Phys. Rev. B 91, 085407 (2015).

[13] X. Liu, J. D. Wood, K.-S. Chen, E. K. Cho, and M. C. Hersam, In situ thermal decomposition of exfoliated two-dimensional black phosphorus, J. Phys. Chem. Lett. 6, 773 (2015).

[14] G. Kresse and J. Furthmüller, Efficient iterative schemes for ab initio total-energy calculations using a plane-wave basis set, Phys. Rev. B 54, 11169 (1996).

[15] G. Kresse and D. Joubert, From ultrasoft pseudopotentials to the projector augmented-wave method, Phys. Rev. B 59, 1758 (1999).

[16] J. P. Perdew, K. Burke, and M. Ernzerhof, Generalized Gradient Approximation Made Simple, Phys. Rev. Lett. 77, 3865 (1996).

[17] H. J. Monkhorst and J. D. Pack, Special points for Brillouin-zone integrations, Phys. Rev. B 13, 5188 (1976).
[18] G. Henkelman, A. Arnaldsson, and H. Jónsson, A fast and robust algorithm for Bader decomposition of charge density, Comput. Mater. Sci. 36, 354 (2006).

[19] M. R. Hestenes and E. Stiefel, Methods of conjugate gradients for solving linear systems, J. Res. Natl. Bur. Stand. 49, 409 (1952).

[20] F. A. Cotton, G. Wilkinson, C. A. Murillo, and M. Bochmann, Advanced Inorganic Chemistry, 6th ed. (Wiley, Hoboken, NJ, 1999).

[21] J. Guan, Z. Zhu, and D. Tománek, Phase Coexistence and Metal-Insulator Transition in Few-Layer Phosphorene: A Computational Study, Phys. Rev. Lett. 113, 046804 (2014).

[22] B. Johansson and N. Mårtensson, Core-level binding-energy shifts for the metallic elements, Phys. Rev. B 21, 4427 (1980).

[23] D. Tomanek, V. Kumar, S. Holloway, and K. H. Bennemann, Semi-empirical theory for surface core-level shifts, Solid State Commun. 41, 273 (1982).

[24] M. Grunze, C. R. Brundle, and D. Tomanek, Adsorption and decomposition of ammonia on a W(110) surface: Photoemission fingerprinting and interpretation of the core level bindingenergies using the equivalent core approximation, Surf. Sci. 119, 133 (1982).

[25] D. Tomanek, P. A. Dowben, and M. Grunze, Thermodynamic interpretation of core-level binding-energies in adsorbates, Surf. Sci. 126, 112 (1983).

[26] T. P. Moffat, R. M. Latanision, and R. R. Ruf, An Xray photoelectron spectroscopy study of chromium-metalloid alloys - III, Electrochim. Acta 40, 1723 (1995).

[27] M. Taniguchi, S. Suga, M. Seki, H. Sakamoto, H. Kanzaki, Y. Akahama, S. Terada, S. Endo, and S. Narita, Valence band and core-level photoemission spectra of black phosphorus single crystals, Solid State Commun. 45, 59 (1983).

[28] Y. Hayasi, T. Takahashi, H. Asahina, T. Sagawa, A. Morita, and I. Shirotani, Electronic structure of black phosphorus studied by polarized soft-x-ray emission and absorption spectroscopy, Phys. Rev. B 30, 1891 (1984).

[29] H. Zhu, X. Qin, A. Azcatl, R. Addou, S. McDonnell, P. D. Ye, and R. M. Wallace, Surface and interfacial study of half cycle atomic layer deposited $\mathrm{Al}_{2} \mathrm{O}_{3}$ on black phosphorus, Microelectron. Eng. 147, 1 (2015).

[30] Y. Harada, K. Murano, I. Shirotani, T. Takahashi, and Y. Maruyama, Electronic structure of black phosphorus studied by x-ray photoelectron spectroscopy, Solid State Commun. 44, 877 (1982). 